\title{
CCL2/CCR2 axis induces hepatocellular carcinoma invasion and epithelial-mesenchymal transition in vitro through activation of the Hedgehog pathway
}

\author{
HUIJIE ZHUANG, GANG CAO, CHANGHUA KOU and TAO LIU \\ Department of Surgical Oncology, Xuzhou Central Hospital, Xuzhou, Jiangsu 221009, P.R. China
}

Received December 23, 2016; Accepted September 12, 2017

DOI: $10.3892 /$ or.2017.6069

\begin{abstract}
Chemokine (C-C motif) ligand 2 (CCL2) has been shown to play an important role in the regulation of tumor cell growth, metastasis and host immune response. CCL2 preferentially binds to the $\mathrm{C}-\mathrm{C}$ chemokine receptor type 2 (CCR2), which is expressed in various tissues. However, the role of the CCL2/CCR2 axis in hepatocellular carcinoma (HCC) invasion and its molecular mechanisms remain unclear. The aim of this study was to elucidate this issue. The human HCC cell line MHCC-97H was treated with CCL2. Cyclopamine, a smoothened (SMO) antagonist, was used to inhibit SMO activity. CCR2 siRNA and Gli-1 siRNA were used to inhibit CCR2 and Gli-1 expression respectively. The effect of CCL2 and Hedgehog (Hh) signaling on cancer cell epithelial-mesenchymal transition (EMT) and invasion was evaluated by quantitative real-time PCR analysis, western blotting and Transwell invasion assay. Our results revealed that CCL2 induced HCC cell invasion and EMT. This effect was accompanied by the activation of Hh signaling, the upregulation of Snail and vimentin and the reduction of E-cadherin. Notably, prior silencing of CCR2 with siRNA abolished CCL2-induced Hh signaling activation, Snail and vimentin upregulation, E-cadherin reduction, as well as HCC cell invasion and EMT. Furthermore, pretreatment with cyclopamine or predepletion of Gli-1 by siRNA also eliminated the changes of Snail, vimentin and E-cadherin, and HCC invasion and EMT caused by CCL2. Collectively, our results revealed that the link between the CCL2/CCR2 axis and the Hh pathway plays an important role in HCC progression. Therefore, the CCL2/CCR2 axis may represent a promising therapeutic target to prevent HCC progression.
\end{abstract}

Correspondence to: Dr Huijie Zhuang, Department of Surgical Oncology, Xuzhou Central Hospital, 199 Liberation of South Road, Xuzhou, Jiangsu 221009, P.R. China

E-mail: zhj2312@163.com

Key words: hepatocellular carcinoma, CCL2/CCR2 axis, Hedgehog signaling, invasion, EMT

\section{Introduction}

Hepatocellular carcinoma (HCC) ranks as the third leading cause of cancer mortality worldwide and the 5-year survival rate of $\mathrm{HCC}$ patients is less than $17 \%$ (1). HCC afflicts nearly 466,100 individuals and causes approximately 422,100 deaths every year in China. Due to the lack of early symptoms and reliable diagnostic markers for early detection, $60 \%$ of patients with HCC are diagnosed at a locally advanced or metastatic stage $(1,2)$. The exact molecular mechanisms which are responsible for this dismal clinical course remain largely unknown. Consequently, it is essential to further elucidate the molecular mechanisms determining HCC metastasis and recurrence.

Chemokines have been shown to play important roles in many aspects of tumor cell biology, including regulation of tumor cell growth, metastasis and host immune response (3). Chemokine (C-C motif) ligand 2 (CCL2), also known as monocyte chemoattractant protein-1 (MCP-1), is a major chemokine that recruits monocytes and macrophages to the sites of inflammation. Recent studies have revealed that a high level of tumor CCL2 expression is associated with unfavorable patient prognosis in various types of cancer (4-6). CCL2 preferentially binds to the $\mathrm{C}-\mathrm{C}$ chemokine receptor type 2 (CCR2), which is expressed in various tissues including thymus, lung, liver, kidney, pancreas and ovary (7). CCL2 has been reported to play an important role in epithelialmesenchymal transition (EMT) and metastasis (8). Targeting tumor-infiltrating macrophages via the CCL2/CCR2 axis was examined as a therapeutic strategy against HCC (9). Shih et al (10) demonstrated that binding of CCL2 to the corresponding CCR2 receptor induced the expression of microRNA-21 and subsequently led to the activation of Rac1 and MMP9 and promoted the migration, invasion and EMT of HCC cells. However, the specific mechanisms and the downstream targets mediated in the CCL2/CCR2-induced invasion of HCC cells remain unclear and need further investigation.

Several studies have demonstrated that sonic Hedgehog $(\mathrm{Hh})$ is overexpressed and plays important roles in many types of cancer, including pancreatic cancer $(11,12)$, breast cancer (13) and HCC (14). The Hh signaling pathway, initiated through the binding of secreted Hh ligands to a 12-pass-transmembrane receptor called Patched 1 (Ptch1), results in smoothened (SMO) dissociation. The activated 
SMO then initiates an intracellular signaling cascade that eventually leads to the activation of the Gli-1 transcription factor and then to the upregulation of the downstream target genes (15). The expression of SMO and Gli-1 is presumed to be a marker of the Hh pathway activation (16). Previous studies have demonstrated that significant hyperactivation of the $\mathrm{Hh}$ signaling has been observed in liver injury and cirrhosis which often lead to the development of HCC lesions (17). The activation of the $\mathrm{Hh}$ pathway in $\mathrm{HCC}$ could induce EMT and thus promote HCC cell invasion and metastasis by upregulating vimentin expression and downregulating E-cadherin expression (18).

In the present study, we focused on the role of the CCL2/CCR2 axis in HCC and the possible mechanisms of the CCL2/CCR2 axis in HCC cell invasion. We found that the activation of CCR2 by its ligand CCL2 increased the expression of SMO and Gli-1, resulting in Hh pathway activation, EMT and HCC cell invasion.

\section{Materials and methods}

Cell culture and reagents. The human $\mathrm{HCC}$ cell line MHCC97H was kindly provided by the Stem Cell Bank of the Chinese Academy of Sciences. The MHCC97H cells were cultured in Dulbecco's modified Eagle's medium (DMEM; Gibco, Grand Island, NY, USA) supplemented with $10 \%$ fetal bovine serum (FBS), $100 \mathrm{U} / \mathrm{ml}$ penicillin and $100 \mu \mathrm{g} / \mathrm{ml}$ streptomycin in a humidified atmosphere of $5 \% \mathrm{CO}_{2}$ at $37^{\circ} \mathrm{C}$. Recombinant human CCL2 was purchased from PeproTech (Rocky Hill, NJ, USA). Cyclopamine was purchased from Selleck Chemicals (Houston, TX, USA). The MHCC97H cells in log phase growth were cultured in 6-well plates in media containing only $1 \%$ FBS for $24 \mathrm{~h}$ before the drug treatment. Subsequently, the drugs were administered at indicated concentrations in medium containing $1 \%$ FBS and the plates were incubated for another $48 \mathrm{~h}$ before a Matrigel invasion assay was performed. The antibodies were purchased from different resources as follows: CCR2-specific rabbit polyclonal antibody (Proteintech, Chicago, IL, USA), Ptch-specific rabbit polyclonal antibody (Proteintech), sonic Hh homolog (SHH)-specific rabbit polyclonal antibody (Proteintech), SMO-specific rabbit polyclonal antibody (Bioworld Technology, Minneapolis, MN, USA), Gli-1-specific mouse monoclonal antibody (Santa Cruz Biotechnology, Dallas, TX, USA), E-cadherin-specific rabbit monoclonal antibody (Cell Signaling Technology, Danvers, MA, USA), Snail-specific rabbit monoclonal antibody (Cell Signaling Technology), vimentin-specific rabbit monoclonal antibody (Cell Signaling Technology) and $\beta$-actin specific mouse monoclonal antibody (Santa Cruz Biotechnology). Horseradish peroxidase-conjugated goat anti-rabbit IgG and goat anti-mouse IgG (Santa Cruz Biotechnology) were used as the secondary antibody.

RNAi transfections. Loss-of-function analysis was performed using siRNAs targeting Gli-1 and CCR 2 which were purchased from GenePharm (Shanghai, China): siRNA against Gli-1 (5'-GGCUCAGCUUGUGUGUAAUTT-3', 5'-AUUACACAC AAGCUGAGCCTT-3'), siRNA againstCCR2 (5'-CTGTCCAC ATCTCGTTCTCGGTTTA-3', 5'-CCCAAAGACCCACTCAT
TTGCAGC-3') and a negative control siRNA (5'-UUCUCCGAA CGUGUCACGUTT-3', 5'-ACGUGACACGUUCGGAGA ATT-3'). The cells were seeded in 6-well plates and were transfected with $100 \mathrm{nM}$ siRNA using Lipofectamine 2000 (Invitrogen; Life Technologies, Carlsbad, CA, USA) according to the manufacturer's instructions. The knockdown of each target gene was confirmed by western blot analysis. The cells were used for subsequent experiments $48 \mathrm{~h}$ after transfection.

Quantitative real-time polymerase chain reaction ( $q R T-P C R$ ) analysis. Total RNA was extracted from the HCC cells using the Fastgen1000 RNA isolation system (Fastgen, Shanghai, China) according to the manufacturer's protocol. Total RNA was reverse-transcribed into cDNA using a PrimeScript RT reagent kit (Takara Bio, Dalian, China). Real-time PCR was used to quantitatively examine the expression of E-cadherin, vimentin, SMO and Gli-1 at the mRNA level. The PCR primer sequences were as follows: for Snail forward, 5'-GGCTCCTT CGTCCTTCTCCT-3' and reverse, 5'-CTGGAGATCCTTG GCCTCAG-3'; for E-cadherin forward, 5'-ATTCTGATTCT GCTGCTCTTG-3' and reverse, 5'-AGTCCTGGTCCTCTTC TCC-3'; for vimentin forward, 5'-CGGGAGAAATTGCAG GAGGA-3' and reverse, 5'-AAGGTCAAGACGTGCCA GAG-3'; for SMO forward, 5'-ACGAGGACGTGGAGGG CTG-3' and reverse, 5'-CGCACGGTATCGGTAGTTCT-3'; for Gli-1 forward, 5'-GGGATGATCCCACATCCTCAGTC-3' and reverse, 5'-CTGGAGCAGCCCCCCCAGT-3'; for $\beta$-actin forward, 5'-AGCGAGTATCCCCCAAAGTT-3' and reverse, 5'-GGGCACGAAGGCTCATCATT-3'. The expression of each target gene was determined using $\beta$-actin as the normalization control. The PCR reactions consisted of $30 \mathrm{sec}$ at $95^{\circ} \mathrm{C}$, followed by 40 cycles at $95^{\circ} \mathrm{C}$ for $5 \mathrm{sec}, 30 \mathrm{sec}$ at $60^{\circ} \mathrm{C}$ and $30 \mathrm{sec}$ at $72^{\circ} \mathrm{C}$. After each qRT-PCR, a dissociation curve analysis was conducted. Relative gene expression was calculated using the $2^{-\Delta \Delta \mathrm{Ct}}$ method (19).

Western blot analysis. Total protein was extracted by RIPA lysis buffer (Beyotime Institute of Biotechnology, Guangzhou, China) and the concentration of protein was determined using the BCA protein assay kit (Pierce, Rockford, IL, USA) according to the manufacturer's instructions. The proteins were subjected to SDS-PAGE using a $10 \%$ polyacrylamide gel with a $5 \%$ stacking gel, and then the protein was transferred to polyvinylidene difluoride (PVDF) membranes. The membranes were initially blocked with $5 \%$ non-fat dry milk in Tris-buffered saline-Tween (TBS-T) for $2 \mathrm{~h}$ and then probed with antibodies against E-cadherin (1:1,000 dilution), Snail (1:1,000 dilution), vimentin (1:1,000 dilution), CCR2 (1:500 dilution), SMO (1:500 dilution), Gli-1 (1:1,000 dilution) or $\beta$-actin (1:1,000 dilution, loading control). After co-incubation with the primary antibodies at $4^{\circ} \mathrm{C}$ overnight, the membranes were blotted with the secondary antibody (1:10,000 dilution) for $2 \mathrm{~h}$ at $37^{\circ} \mathrm{C}$. Chemiluminescence detection of bound antibodies was performed using an enhanced chemiluminescence ECL Plus system and a Molecular Imager ChemiDoc XRS system (Bio-Rad Laboratories, Hercules, CA, USA).

Cell viability assay. The MHCC $97 \mathrm{H}$ cells were plated into 96-well plates at a density of 5,000 cells/well and allowed to adhere for at least $24 \mathrm{~h}$ and serum-starved overnight 
B

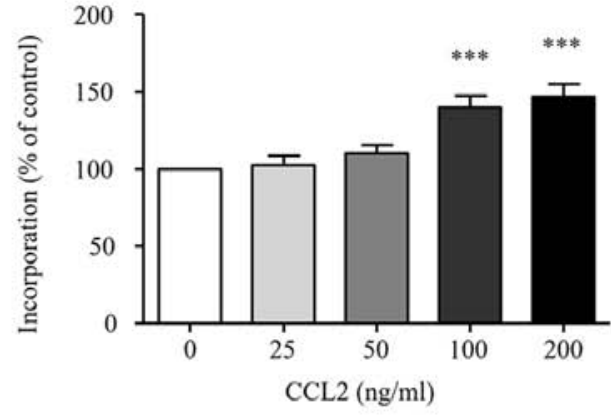

C

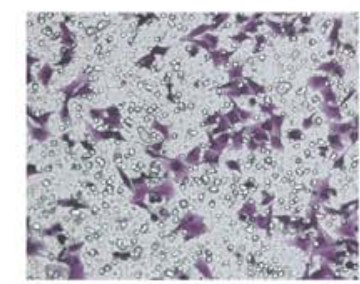

$\operatorname{CCL} 2(\mathrm{ng} / \mathrm{ml})$

0

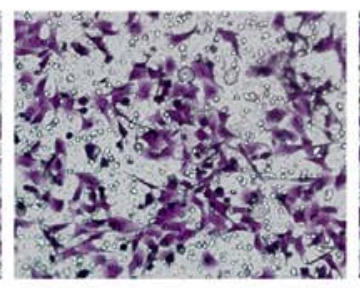

50

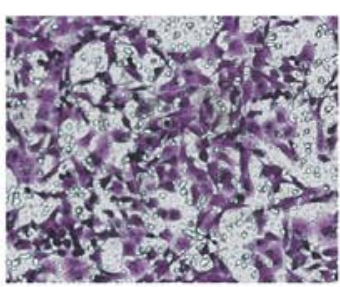

100

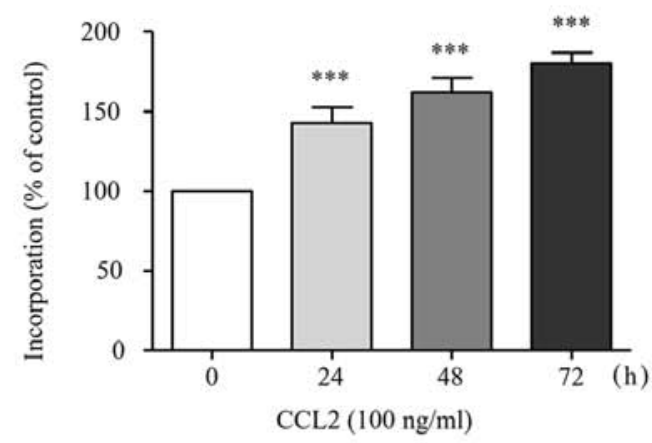

D

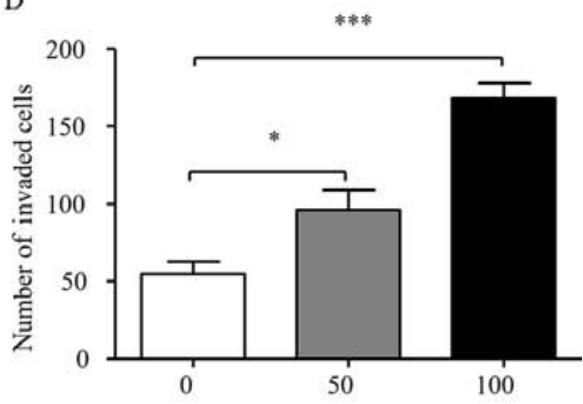

Figure 1. The effect of CCL2 on the proliferation and invasion ability of human HCC cells. (A) The HCC MHCC97H cells were stimulated with different concentrations of CCL2, ranging from 0 to $200 \mathrm{ng} / \mathrm{ml}$ for $24 \mathrm{~h}$ and the percentage of incorporation in the cells was determined by an MTT assay. (B) The cells were exposed to $100 \mathrm{ng} / \mathrm{ml} \mathrm{CCL2}$ for the indicated time-points and the percentage of incorporation in the cells was assessed by an MTT assay. MHCC97H cells in DMEM containing 1\% FBS were seeded in the Matrigel-coated Transwell upper chambers. FBS (10\%) was used as a chemoattractant. After $24 \mathrm{~h}$, the cells on the upper surface of the filters were removed and then the filters were stained with crystal violet. (C) Representative staining, x200 magnification. (D) The number of invaded cells was quantified by counting the cells from 5 random fields at $x 200$ magnification. The data are representative of 3 independent experiments. Column, mean ( $\mathrm{n}=3)$; bar, $\mathrm{SD} ;{ }^{*} \mathrm{P}<0.05,{ }^{* * *} \mathrm{P}<0.001$. CCL2, chemokine (C-C motif) ligand 2; HCC, hepatocellular carcinoma.

(1\% FBS in DMEM) before the beginning of the experiments. After different treatments, the cell viability was assessed by the 3-(4,5-dimethylthiazol-2-yl)-2,5-diphenyltetrazolium bromide (MTT) assay. Twenty microliters of $5 \mathrm{mg} / \mathrm{ml}$ MTT solution per $100 \mu \mathrm{l}$ of growth medium was added into each well after the removal of the media and incubation followed at $37^{\circ} \mathrm{C}$ for $4 \mathrm{~h}$. Following, $100 \mu \mathrm{l}$ DMSO was added to each well and the optical density (OD) was assessed at $490 \mathrm{~nm}$ on a multifunction microplate reader (POLARstar OPTIMA; BMG Labtech Ltd., Ortenberg, Germany). The proliferation rate was calculated according to the following equation: proliferation rate $=(\mathrm{OD}$ sample $/ \mathrm{OD}$ control $) \times 100 \%$.

Cell invasion analysis. The MHCC97H cell invasion was assessed by a chamber-based invasion assay. In brief, the upper surface of a filter (pore size, $8.0 \mu \mathrm{m}$; Millipore, Billerica, MA, USA) was coated with basement membrane Matrigel (BD Biosciences, Franklin Lakes, NJ,USA). The cells were suspended in DMEM containing $1 \%$ FBS. Then the cell suspensions $(200 \mu \mathrm{l}$ containing 40,000 cells) were added to the upper chambers. Simultaneously, $500 \mu \mathrm{l}$ of DMEM containing 10\% FBS was placed in the lower chambers. The cells were allowed to migrate for $24 \mathrm{~h}$ at $37^{\circ} \mathrm{C}$. The non-invading cells were removed from the upper surface by scraping with a wet cotton swab. After rinsing with phosphate buffered saline (PBS), the filter was fixed and stained with crystal violet. The invasion ability was determined by counting the stained cells on the bottom surface. Five random fields were captured at a magnification, $x 200(n=3)$.
Immunofluorescence staining. The cells were fixed in $4 \%$ formaldehyde diluted in PBS for $15 \mathrm{~min}$, permeabilized with $0.3 \%$ Triton X-100, treated with blocking buffer (5\% BSA in PBS), and then incubated overnight with the primary antibody at $4^{\circ} \mathrm{C}$. The cells were then incubated with Red-conjugated secondary antibody from Jackson Immunoresearch Laboratories (West Grove, PA, USA) for $1 \mathrm{~h}$ at room temperature. Slides were mounted and examined using a Zeiss Instruments microscope (Carl Zeiss, Oberkochen, Germany).

Statistical analysis. Statistical analysis was performed using SPSS 17.0 software (SPSS, Inc., Chicago, IL, USA). Each experiment was performed at least three times. Data are presented as the means \pm standard deviation (SD). Differences between the groups were analyzed by analysis of variance (ANOVA). $\mathrm{P}<0.05$ was considered to indicate a statistically significant difference.

\section{Results}

CCL2 promotes the invasion of HCC cells via the induction of EMT. The MHCC $97 \mathrm{H}$ cells were treated with different concentrations of $\operatorname{CCL} 2(0,25,50,100$ and $200 \mathrm{ng} / \mathrm{ml})$ at different time-points $(24,48$ and $72 \mathrm{~h})$ and the cell proliferation was assessed by an MTT assay. Fig. 1A indicates that CCL2 stimulated MHCC97H proliferation in a dose-dependent manner. As shown in Fig. 1B, CCL2 time-dependently stimulated $\mathrm{MHCC} 97 \mathrm{H}$ proliferation, indicating that CCL2 
A

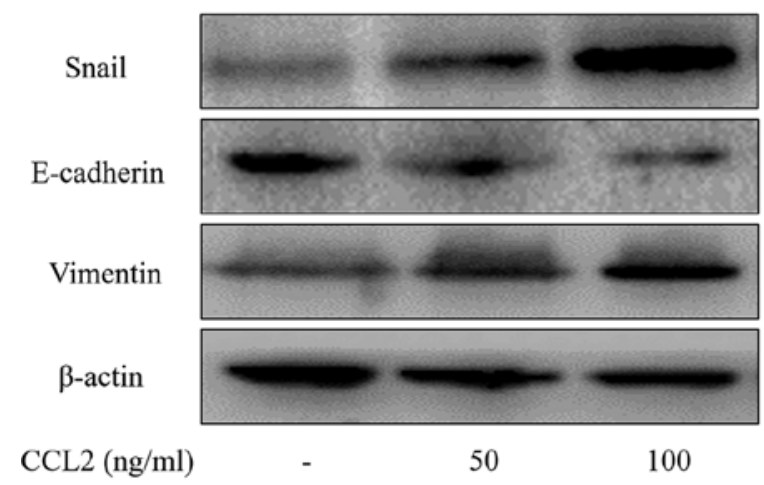

$\mathrm{C}$

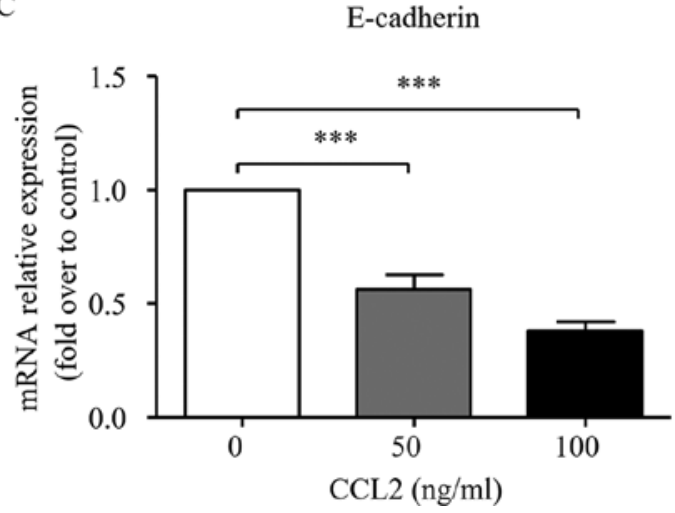

B

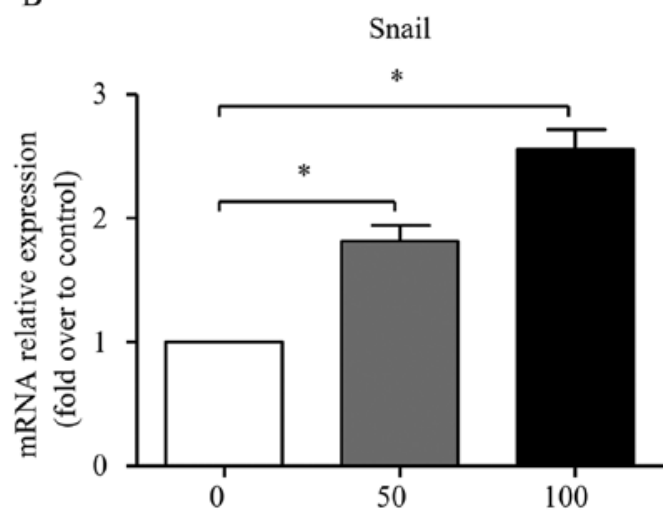

D

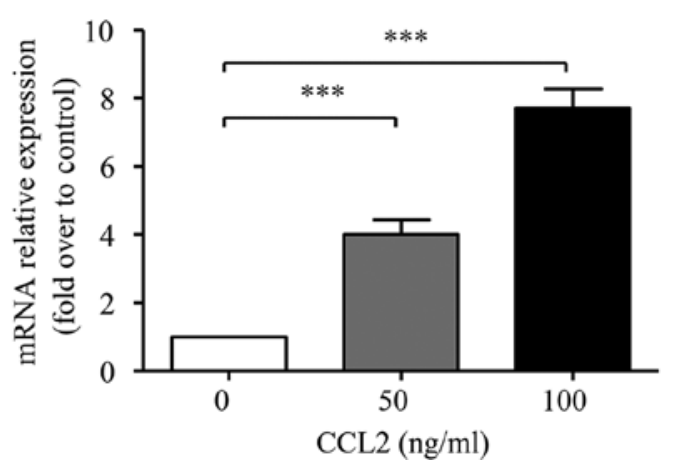

Figure 2. Effect of CCL2 on EMT of human HCC cells. The HCC MHCC97H cells were treated with 50 or $100 \mathrm{ng} / \mathrm{ml}$ CCL2 for $48 \mathrm{~h}$. (A) Whole cell protein extracts were subjected to western blot analysis using Snail, E-cadherin and vimentin antibodies. $\beta$-actin was used as an internal loading control. (B-D) Total RNA was extracted and the expression of Snail, E-cadherin and vimentin was assessed by real-time RT-PCR. The expression of each target gene was quantified using $\beta$-actin as a normalization control. The data represent the results from three independent experiments. Column, mean ( $\mathrm{n}=3$ ); bar, $\mathrm{SD} ;{ }^{*} \mathrm{P}<0.05$, ${ }^{* * *} \mathrm{P}<0.001$. CCL2, chemokine (C-C motif) ligand 2; EMT, epithelial-mesenchymal transition; HCC, hepatocellular carcinoma.

could effectively stimulate MHCC97H proliferation. These findings were consistent with the results that CCL2 promoted non-small cell lung cancer A549 cell viability (20). The MHCC97H cells were treated with or without CCL2 for $24 \mathrm{~h}$ and then the invasion ability was assessed via a Transwell assay. The results demonstrated that 50 or $100 \mathrm{ng} / \mathrm{ml}$ of CCL2 significantly increased the invasion ability of $\mathrm{MHCC} 97 \mathrm{H}$ cells $(\mathrm{P}<0.05$, Fig. $1 \mathrm{C}$ and $\mathrm{D})$. These results reveal that CCL2 promotes cell invasion.

EMT has been confirmed to play an important role in cancer progression, which is characterized by the loss of cell-cell adhesion with diminished expression of epithelial markers such as E-cadherin and increased expression of mesenchymal markers such as vimentin and EMT transcription factors such as Snail (21). To further investigate the possible role of the CCL2/CCR2 axis on EMT process, the expression of Snail, E-cadherin and vimentin was detected via western blot analysis and real-time PCR at both the protein and mRNA levels. As shown in Fig. 2, both 50 and $100 \mathrm{ng} / \mathrm{ml}$ of CCL2 significantly decreased the expression of E-cadherin at both the mRNA and protein levels. Simultaneously, the expression levels of Snail and vimentin at both the mRNA and protein levels were significantly increased after the CCL2 treatment.

CCL2 promotes the invasion ability and EMT of HCC cells in a CCR2-dependent manner. Although CCL2 influenced the EMT process and cell invasive ability, the underlying mechanisms remain largely unclear. CCR2 knockdown was carried out in the present study to investigate the role of CCR2 in CCL2-induced HCC invasion. Transfection of CCR 2 siRNA significantly reduced CCR2 expression (Fig. 3A). Transfection of CCR2 siRNA reversed the effect of CCL2 on MHCC97H cell invasion $(\mathrm{P}<0.05$; Fig. 3B and $\mathrm{C})$. Furthermore, the CCL2-induced downregulation of E-cadherin and the upregulation of Snail and vimentin in HCC cells were abolished upon knockdown of CCR2 (Fig. 3D-G). Thus, these data reveal that CCL2 promotes HCC progression through its receptor CCR2.

CCL2 promotes the activation of the Hh pathway in HCC cells in a CCR2-dependent manner. Several studies have revealed that the Hh pathway may be a treatment target for HCC $(14,22)$. Our results revealed that CCL2 significantly increased the transcription of the Hh pathway-related genes, including SMO and Gli-1 in MHCC97H cells. However, the levels of SHH and Ptch remained unchanged, compared to the normal controls (Fig. 4A-C). CCR2 knockdown was carried out in the present study, to investigate the role of CCR2 in CCL2-induced activation of the Hh pathway. Transfection of CCR2 siRNA significantly decreased the CCL2-induced expression of SMO and Gli-1 both at the protein and mRNA levels, but there was no effect on Ptch and SHH expression levels (Fig. 4D-F). These results indicated that Hh signaling was 
A

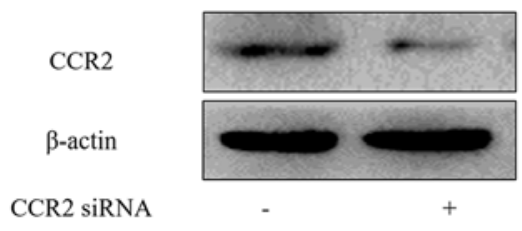

C

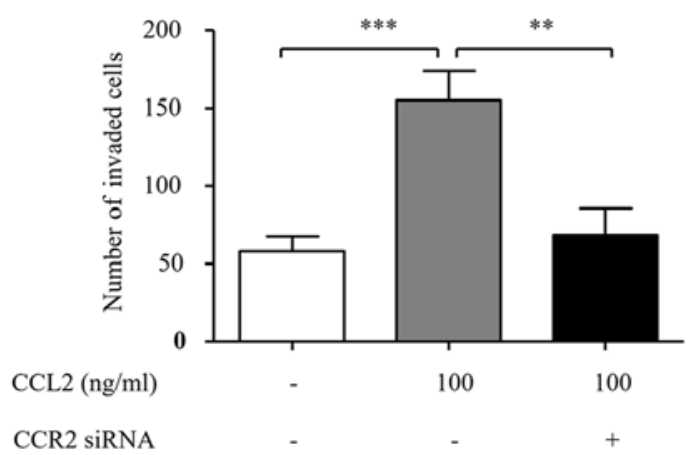

E

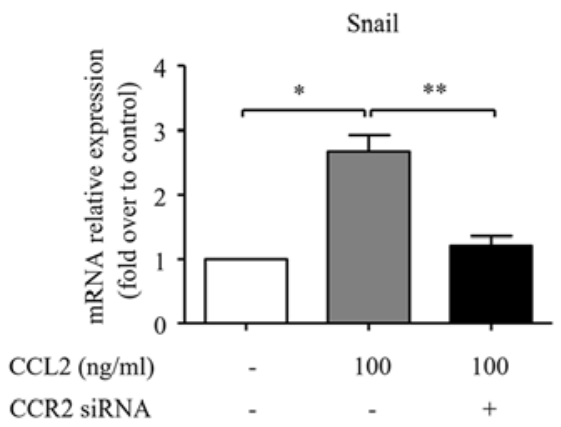

B

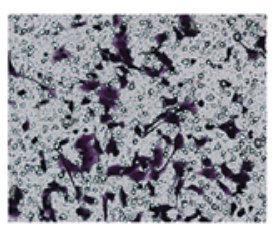

CCL2 (ng/ml)

-

CCR2 siRNA

D

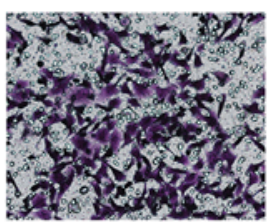

100
100

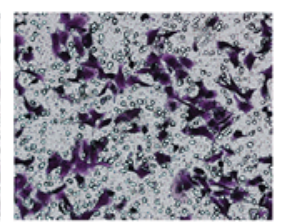

$+$

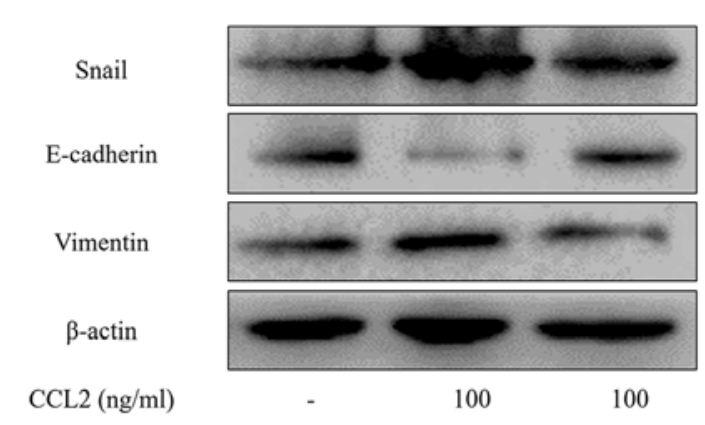

CCR2 siRNA

F

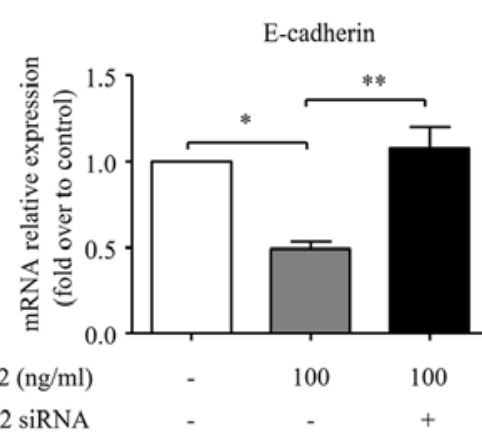

G

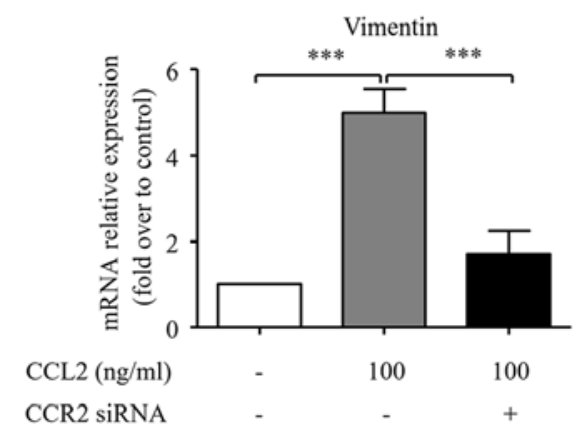

Figure 3. CCR2 interference reverses CCL2-induced EMT and invasion. (A) Knockdown of CCR2 by siRNA for $48 \mathrm{~h}$ was confirmed by western blot analysis. (B) The HCC MHCC97H cells were transfected with CCR2 siRNA for $48 \mathrm{~h}$ and then treated with CCL2. The number of cells was assessed under a light microscope. (C) The number of invaded cells was quantified by counting the cells from 5 random fields at x200 magnification. The data are representative of 3 independent experiments. (D) The Snail, E-cadherin and vimentin protein levels were assessed via western blot analysis. (E-G) The Snail, E-cadherin and vimentin mRNA levels were analyzed by real-time RT-PCR. Column, mean ( $\mathrm{n}=3)$; bar, SD; ${ }^{*} \mathrm{P}<0.05,{ }^{* * *} \mathrm{P}<0.01,{ }^{* * *} \mathrm{P}<0.001$. CCR2, C-C chemokine receptor type 2; CCL2, chemokine (C-C motif) ligand 2; EMT, epithelial-mesenchymal transition.

activated in MHCC97H cells under the CCL2 treatment. Upon activation, Gli-1 proteins translocate from the cytoplasm to the nucleus and activate the transcription of target genes $(23,24)$. Additionally, as demonstrated by immunofluorescence, the nuclear translocation of Gli-1 was enhanced as an effect of CCL2 treatment, while transfection of CCR2 siRNA obviously decreased the nuclear translocation of Gli-1 (Fig. 4G). Collectively, these findings indicate that CCL2 activates the Hh pathway in a CCR2-dependent manner.

SMO mediates CCL2-induced EMT and cell invasion in HCC cells. Since the activation of the CCL2/CCR2 axis simultaneously induces HCC cell invasion and Hh pathway activation, we hypothesized that there is a cross-talk between the CCL2/CCR2 axis and the Hh pathway and this cross-talk contributes to HCC cell invasion. To test this hypothesis, we investigated the relationship between the
CCL2/CCR2-induced cell invasion and Hh pathway activation using an SMO antagonist, cyclopamine, as an inhibitor of the Hh pathway (25). Notably, pretreatment with cyclopamine significantly decreased HCC cell invasion (Fig. 5A and B), reversed the downregulation of E-cadherin and the upregulation of Snail and vimentin and reduced the expression of SMO and Gli-1 even in the presence of CCL2 (Fig. 5C). The Snail, SMO, Gli-1, E-cadherin and vimentin mRNA levels were consistent with the protein changes (Fig. 5D-H). In addition, immunofluorescence staining of these treated cells also ascertained that CCL2 induced Gli-1 expression in the nucleus of $\mathrm{MHCC} 97 \mathrm{H}$ cells, while pretreatment with cyclopamine markedly decreased the nuclear translocation of Gli-1 (Fig. 5I). Therefore, we concluded that the inhibition of the Hh pathway activation via blocking the function of SMO eliminates cell invasion and EMT induced by CCL2. These findings reveal that there is a cross-talk between the 
A

SHH

Ptch

Gli-1

SMO

$\beta$-actin

CCL2 (ng/ml)

$\mathrm{D}$

SHH

Gli-1

SMO

$\beta$-actin

CCL2 (ng/ml)

CCR2 siRNA

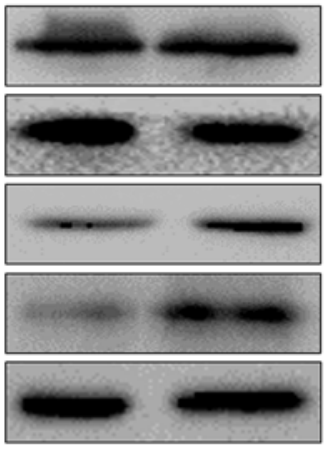

100
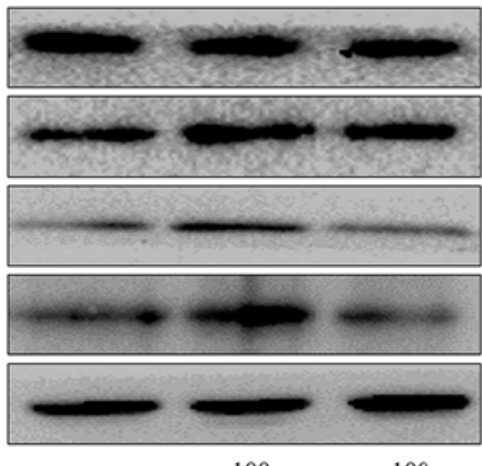

100

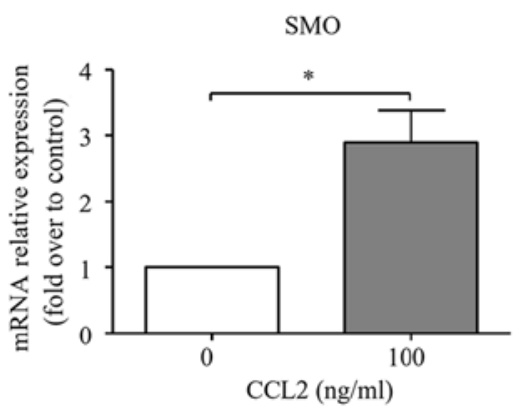

$\mathrm{C}$

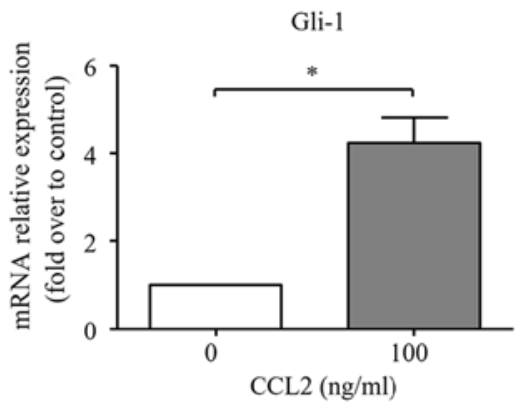

E

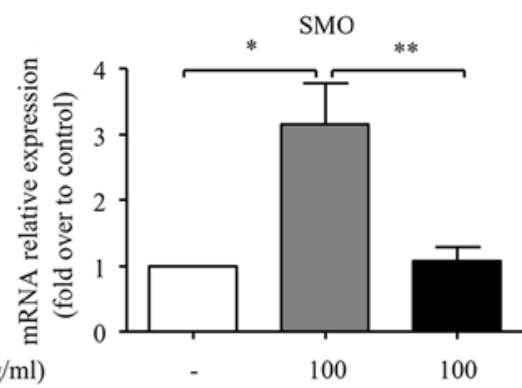

CCL2 (ng/ml)

CCR2 siRNA

\section{G}
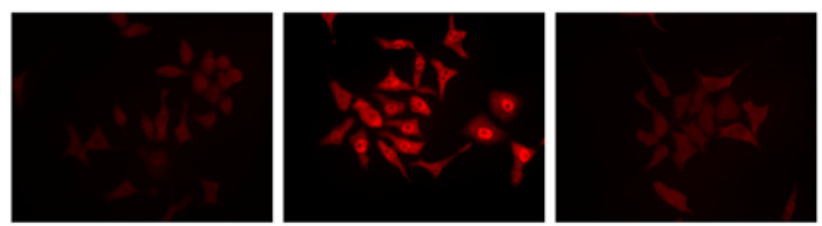

$\mathrm{F}$

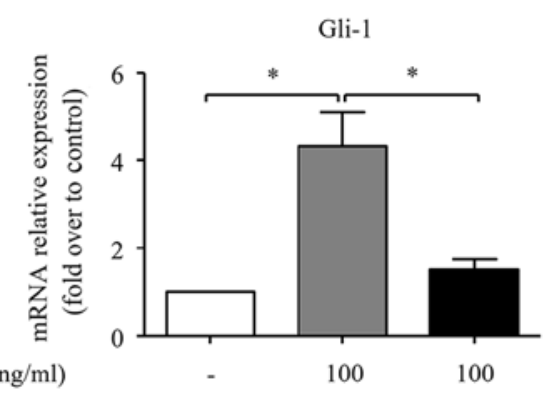

DAPI
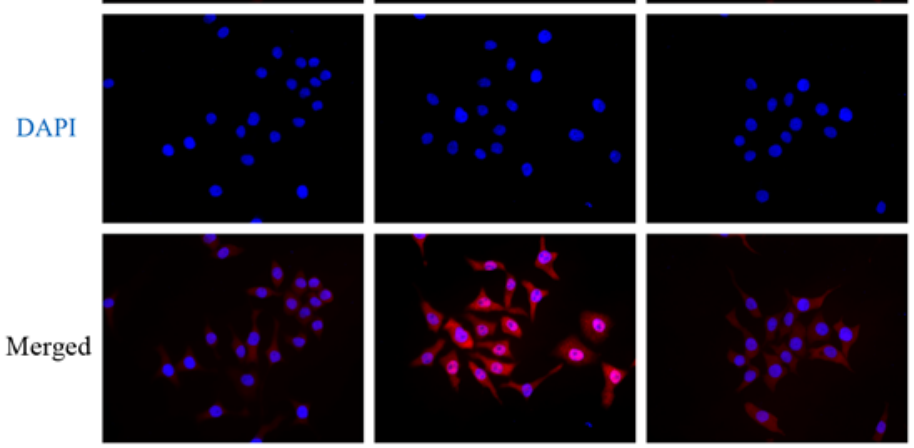

100

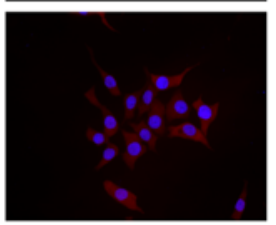

CCL2 (ng/ml)

100

CCR2 siRNA

Figure 4. CCL2/CCR2 axis activates the Hh pathway in human HCC cells. (A) The expression of SHH, Ptch, SMO and Gli-1 proteins in the MHCC97H cells was evaluated by western blot analysis following treatment with the CCR2 ligand CCL2 for $48 \mathrm{~h}$. (B and C) The expression of SMO and Gli-1 at mRNA levels was assessed by real-time RT-PCR for the same cells. (D) CCR2 siRNA diminished the effects of CCL2 on the expression of Ptch, SHH, SMO and Gli-1 at the protein level in MHCC97H cells, as determined by western blot analysis. (E and F) CCR2 siRNA diminished the effects of CCL2 on the expression of SMO and Gli-1 at mRNA level in the MHCC97H cells, as determined by real-time RT-PCR. (G) Immuofluorescence staining of Gli-1 in the MHCC97H cells under normal control or $100 \mathrm{ng} / \mathrm{ml} \mathrm{CCL2} \mathrm{stimulation} \mathrm{or} \mathrm{CCR2} \mathrm{siRNA} \mathrm{combined} \mathrm{with} 100 \mathrm{ng} / \mathrm{ml} \mathrm{CCL2} \mathrm{stimulation} \mathrm{for} 48 \mathrm{~h}$. Red represents Gli-1 staining. Blue represents nuclear DNA staining by DAPI. Column, mean $(\mathrm{n}=3)$; bar, SD; ${ }^{*} \mathrm{P}<0.05 ;{ }^{* *} \mathrm{P}<0.01$. CCL2, chemokine (C-C motif) ligand 2; CCR2, C-C chemokine receptor type 2 ; Hh, Hedgehog; HCC, hepatocellular carcinoma; SHH, sonic Hh homolog; Ptch, Patched 1; SMO, smoothened.

CCL2/CCR2 axis and the Hh pathway activation, which is mediated by an increased SMO expression.

Gli-1 mediates CCL2-induced EMT and cell invasion in HCC cells. Previous studies have demonstrated that Gli-1 induces mobility and invasion of HCC cells and is an important regulator of EMT (18). In the present study, since we found that the activation of CCR 2 by CCL 2 simultaneously induces tumor cell invasion and Hh pathway activation, we hypothesized that Gli-1 is a critical mediator of the CCL2-induced EMT and cell invasion of HCC cells. In order to further confirm the effect of Gli-1 on CCL2-induced invasion of HCC cells, we investigated the role of Gli-1 in the CCL2/CCR2 axis-induced invasion and $\mathrm{Hh}$ pathway activation using siRNA targeting Gli-1. Transfection of Gli-1 siRNA significantly reduced the Gli-1 protein level (Fig. 6A). Furthermore, Transwell assays revealed that transfection with Gli-1 siRNA significantly inhibited the invasion ability of HCC cells induced by CCL2 (Fig. 6B and C). The knockdown of Gli-1 blocked the CCL2-induced increase in Snail and vimentin and reduction 
A

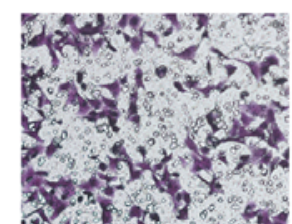

CCL2 (ng/ml)

Cyc $(10 \mu \mathrm{M})$

$\mathrm{C}$

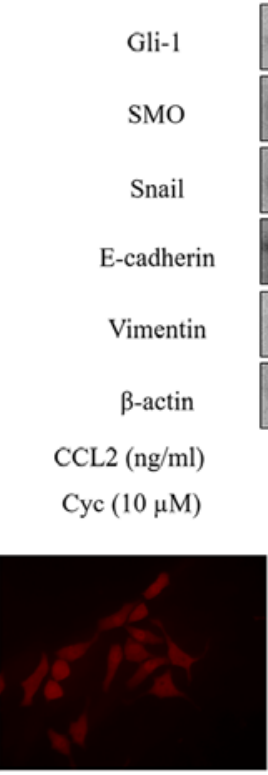

DAPI
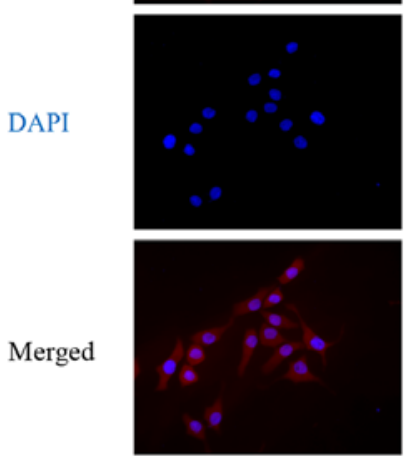

CCL2 (ng/ml)

Cyc $(10 \mu \mathrm{M})$

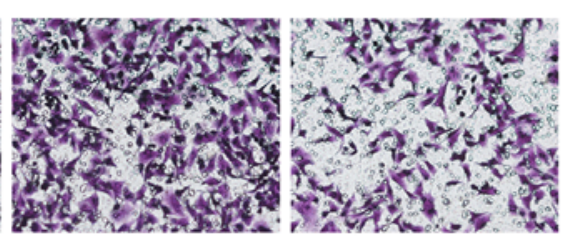

100

100

$+$
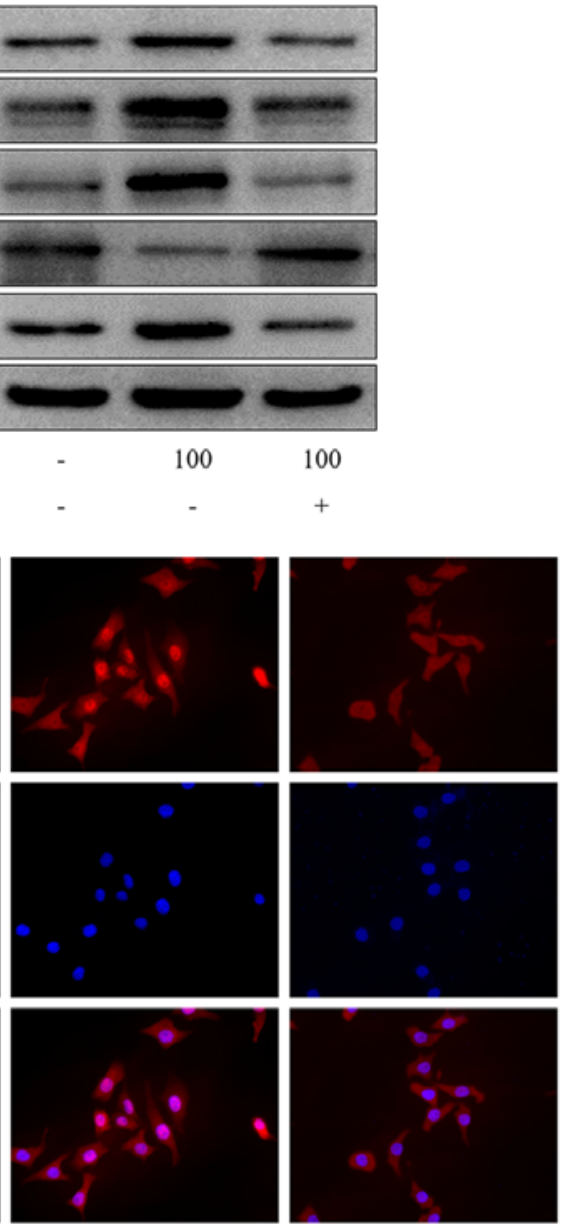

100
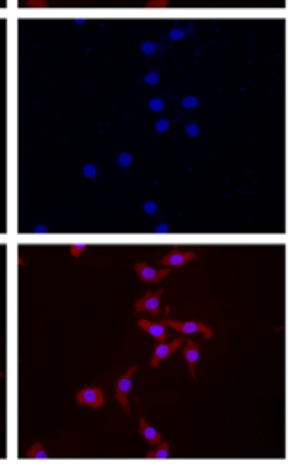

B

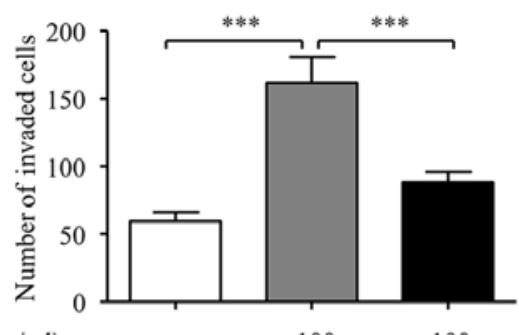

$\operatorname{CCL} 2(\mathrm{ng} / \mathrm{ml})$

100

100

D Cyc $(10 \mu \mathrm{M})$
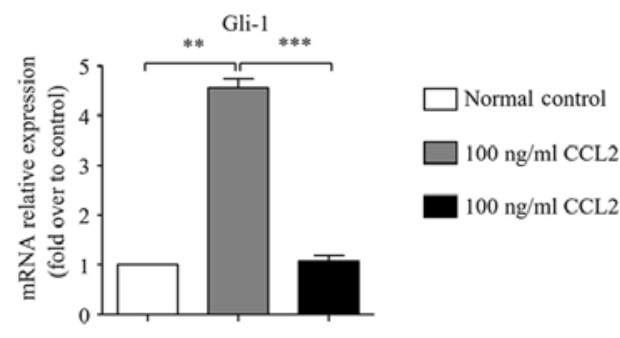

$100 \mathrm{ng} / \mathrm{ml} \mathrm{CCL} 2+10 \mu \mathrm{M} \mathrm{Cyc}$
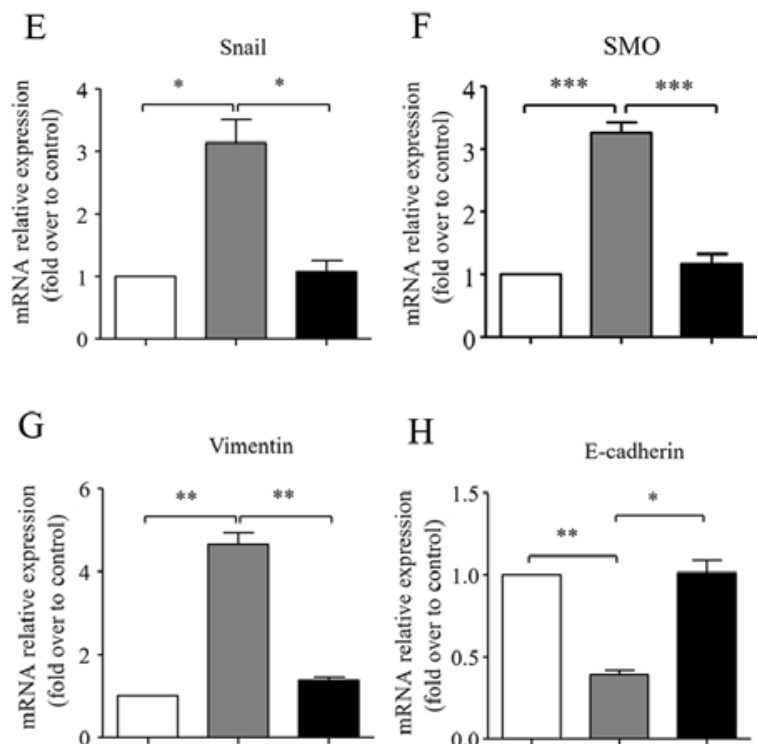

Figure 5. The effects of Cyc on CCL2-induced HCC cell invasion and EMT. (A) The effects of Cyc (10 $\mu \mathrm{M})$ on HCC cell invasion. After treatment with Cyc for $24 \mathrm{~h}$, the cells were seeded into a Matrigel-coated invasion chamber with or without CCL2 for $24 \mathrm{~h}$. The number of cells was counted under a light microscope. (B) The number of invaded cells was quantified by counting the cells from 5 random fields at $x 200$ magnification. The data are representative of 3 independent experiments. (C) The EMT-related molecules Snail, E-cadherin and vimentin, and the Hh pathway-related proteins SMO and Gli-1 protein levels were measured via western blot analysis. (D-H) The EMT-related molecules Snail, E-cadherin and vimentin, and the Hh pathway-related proteins SMO and Gli-1 mRNA levels were analyzed by real-time RT-PCR. (I) Immuofluorescence staining of Gli-1 in MHCC97H cells under normal control or 100 ng/ml CCL2 stimulation or $10 \mu \mathrm{M}$ Cyc combined with $100 \mathrm{ng} / \mathrm{ml} \mathrm{CCL2}$ stimulation for $48 \mathrm{~h}$. Red represents Gli-1 staining. Blue represents nuclear DNA staining by DAPI. Column, mean ( $\mathrm{n}=3)$; bar, $\mathrm{SD} ;{ }^{*} \mathrm{P}<0.05,{ }^{* *} \mathrm{P}<0.01,{ }^{* * *} \mathrm{P}<0.001$. Cyc, cyclopamine; CCL2, chemokine (C-C motif) ligand 2; HCC, hepatocellular carcinoma; EMT, epithelial-mesenchymal transition; Hh, Hedgehog; SMO, smoothened.

of E-cadherin protein levels (Fig. 5D). These data demonstrate that CCL2 promotes HCC invasion and EMT by modulating Gli-1-dependent Snail, vimentin and E-cadherin expression.

\section{Discussion}

Chemokines are a family of small, secreted proteins that have pleiotropic roles in inflammation-related pathological diseases, including cancer. CCL2 is broadly expressed in a variety of tissue types and acts as a potent chemoattractant to recruit monocytes and macrophages to the sites of inflammation. Recent studies have revealed that a high expression of CCL2 is associated with unfavorable patient prognosis in various types of cancer (4-6). CCL2 is overexpressed in human liver cancer and is an independent prognostic indicator in patients with HCC. Blockade of the CCL2/CCR2 axis by knockdown 
A

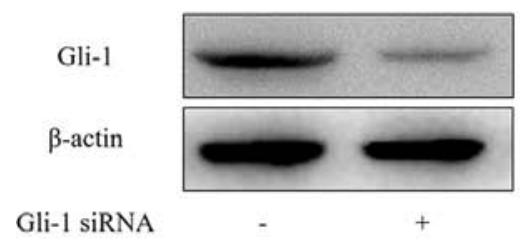

B

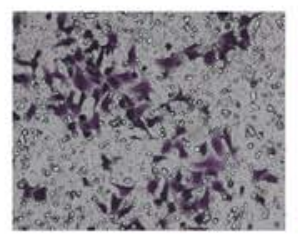

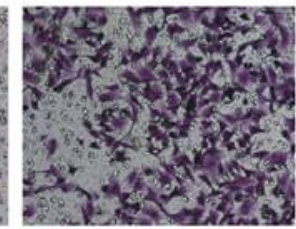

100

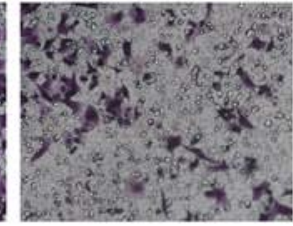

$-$

si-Gli-1

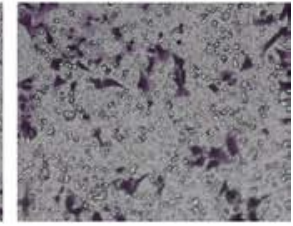

100 siRNA si-control

si-control

si-Gli-1

$\mathrm{C}$

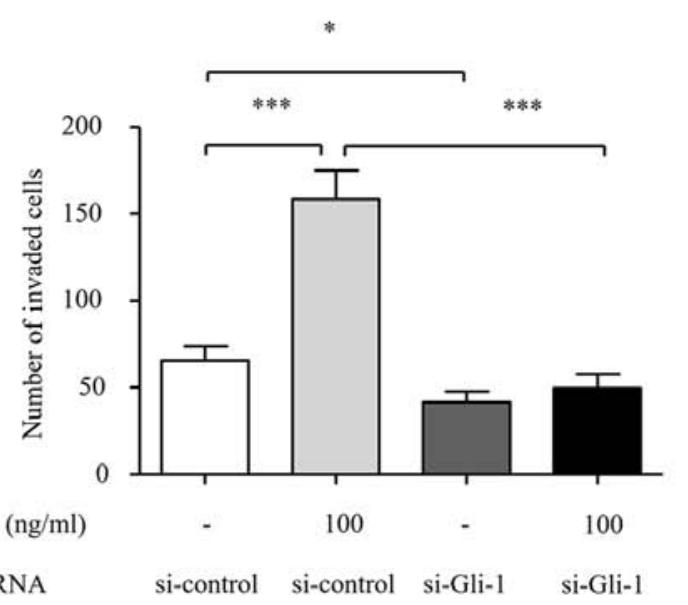

$\mathrm{D}$

Snail
Vimentin
E-cadherin
Gli-1
SMO
$\beta$-actin
CCL2 (ng/ml)
siRNA

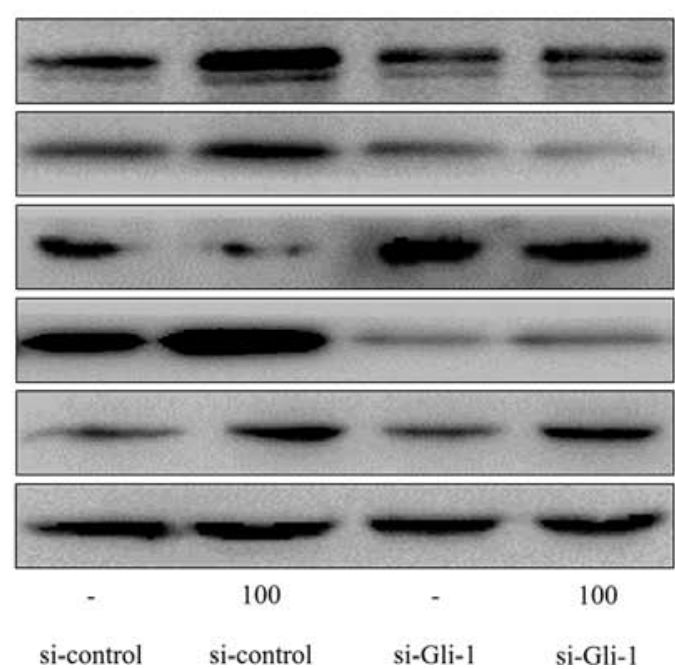

Figure 6. Gli-1 siRNA abolishes the effects of CCL2-mediated invasion and EMT in HCC cells. (A) Knockdown of Gli-1 by siRNA for $48 \mathrm{~h}$ was confirmed by western blot analysis. (B) The effect on cell invasion in response to Gli-1 knockdown. After transfection with siRNA for $48 \mathrm{~h}$, the cells were seeded into a Matrigel-coated invasion chamber with or without CCL2 for $24 \mathrm{~h}$. The number of cells was counted under a light microscope. (C) The number of invaded cells was quantified by counting the cells from 5 random fields at x 200 magnification. The data are representative of 3 independent experiments. (D) The effects of Gli-1 siRNA on the expression of Snail, SMO, Gli-1, E-cadherin and vimentin. After transfection of the cells with siRNA for $48 \mathrm{~h}$, Snail, SMO, Gli-1, E-cadherin and vimentin expression levels were determined by western blot analysis. Column, mean ( $\mathrm{n}=3)$; bar, SD; ${ }^{*} \mathrm{P}<0.05$, ${ }^{* * *} \mathrm{P}<0.01,{ }^{* * *} \mathrm{P}<0.001$. CCL2, chemokine (C-C motif) ligand 2; EMT, epithelial-mesenchymal transition; HCC, hepatocellular carcinoma; SMO, smoothened.

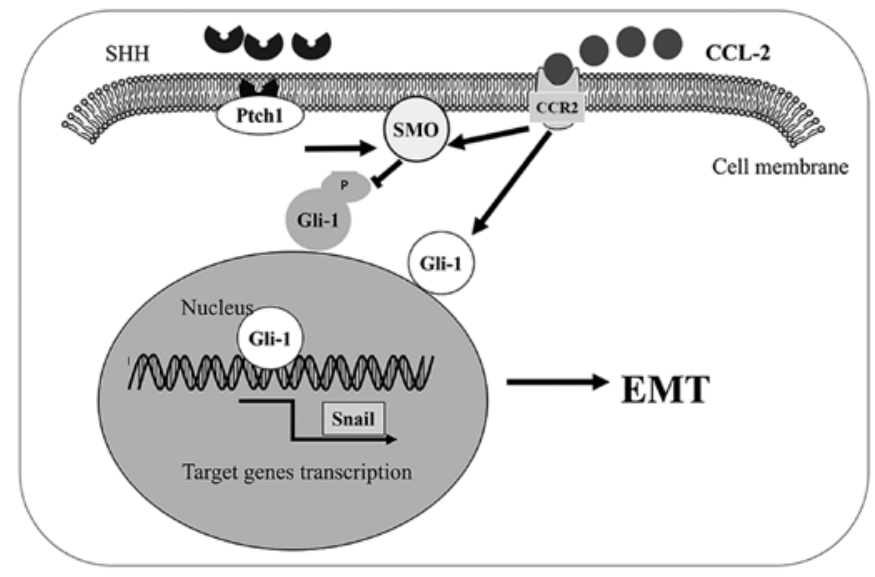

Figure 7. Schematic diagram of the CCL2/CCR2 axis activating Hh signaling. CCL2 triggers SMO and Gli-1 expression. Accumulated Gli-1 translocates to the nucleus, results in the activation of the EMT-related genes, leading to EMT and invasion of HCC cells. CCL2, chemokine (C-C motif) ligand 2; CCR2, C-C chemokine receptor type 2; Hh, Hedgehog; SMO, smoothened; EMT, epithelial-mesenchymal transition; HCC, hepatocellular carcinoma.

of CCR2 or with a CCR2 antagonist inhibits malignant growth and metastasis, reduces postsurgical recurrence and enhances survival (9). CCL2 is secreted by different cell types and is involved in various aspects of liver pathogenesis, including acute liver injury, chronic $\mathrm{HBV} / \mathrm{HCV}$ infection, cirrhosis and tumorigenesis (26-28). CCR2, the only known receptor for CCL2, has been found to regulate cell growth, angiogenesis, invasion and metastasis (29). However, the role of the CCL2/CCR2 axis in HCC cell invasion and its molecular mechanisms remain poorly understood. Our results revealed that CCL2 promotes HCC invasion in a dose-dependent manner.

EMT occurs as a key step during embryonic morphogenesis and is now implicated in the progression of primary tumors towards metastases. In recent years, numerous studies have ascertained that EMT plays important roles in the progression of human cancer (30). The zinc-finger transcriptional repressor Snail reportedly contributes to EMT in HCC and plays a key role in tumorigenesis, differentiation, migration and invasiveness (31). In addition, recent research has revealed that Snail expression is negatively related to tumor differentiation, which is an independent factor predictive of survival in HCC patients (32). Loss of E-cadherin and gain of vimentin are known to play key roles in the EMT process of various human types of cancer, including HCC (18). Previous studies have demonstrated that CCL2 induces this phenomenon via 
the EMT-related genes $(8,33)$. In the present study, our results revealed that CCL2 induced upregulation of the EMT transcription factor Snail and the mesenchymal marker vimentin, however, the epithelial marker E-cadherin was downregulated after treatment with CCL2 (Fig. 2). However, the EMT process and invasion ability were reversed after transfection with CCR2 siRNA, which indicated that the CCL2-induced HCC invasion and EMT are CCR2 receptor-dependent (Fig. 3).

Several studies have demonstrated that multiple components of Hh signaling are affiliated with EMT, invasion and metastasis in cancer cells (34-37). In the present study, our results indicated that CCL2 significantly induced SMO and Gli-1 expression. The immunofluorescence staining results also confirmed that CCL2 induced Gli-1 translocation into the nucleus of MHCC97H cells. However, $\mathrm{Ptch}$ and $\mathrm{SHH}$ remained unchanged compared to normal controls. Based on previously published studies and our findings, we thus hypothesized that a cross-talk exists between the CCL2/CCR2 axis and the Hh pathway, which is critical for CCL2-induced cancer cell invasion and EMT. This hypothesis is further supported by the fact that knockdown of CCR 2 by siRNA prevented the activation of the Hh pathway and the invasion by CCL2, suggesting that CCR2 is the key factor in the CCL2-mediated activation of the Hh pathway and pro-invasion ability.

The Hh signaling pathway, which is normally quiescent in adult liver, has been shown to be very active in HCC (38). Without binding to Hh ligands, Ptch holds SMO, a seven transmembrane spanning protein, in an inactive state and thus prohibits signaling to downstream genes. Once activated, SMO initiates the transcriptional activity of downstream targets and Gli-1 proteins translocate from the cytoplasm to the nucleus and activate the transcription of target genes, which in turn regulate cell proliferation, differentiation, apoptosis and invasion (39-41). Therefore, the expression of SMO and Gli-1 is presumed to be a marker of the Hh pathway activation.

Cyclopamine, an SMO antagonist, especially binds to SMO heptahelical bundle to inhibit its activity so as to suppress $\mathrm{Hh}$ signaling. We exposed HCC cells to cyclopamine in the presence of CCL2. Cyclopamine markedly reduced the tumor invasion and reversed the EMT progress induced by CCL2. Consistent with previous results, blocking SMO function with cyclopamine decreased the expression of the transcription factor Gli-1. We also observed that the nuclear Gli-1 expression was downregulated via immunofluorescence (Fig. 5). It is known that Gli-1 is a critical mediator of the Hh pathway in cancer cell invasion and metastasis, which was consistent with our results that Gli-1 siRNA inhibited EMT progress by suppressing Snail and vimentin expression and enhancing E-cadherin expression. Subsequently we treated the HCC cell lines with siRNA specific to Gli-1 in the presence of CCL2. Prior silencing of Gli-1 with siRNA abolished CCL2-induced Snail and vimentin upregulation, E-cadherin reduction, as well as HCC invasion and EMT. However, Gli-1 siRNA could not interrupt the CCL2-mediated increase in SMO. Collectively, these findings reveal that it is probable that CCL2 induced EMT and cell invasion via the activation of SMO and Gli-1 expression (Fig. 6).

In some preclinical cancer models, targeting of CCL2 is an effective therapeutic approach. The neutralizing antibody carlumab (formerly named CNTO 888) is a human immuno-

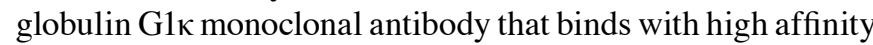

and specificity to human CCL2 and has entered clinical trials for the treatment of prostate cancer (42). Li et al findings (9) revealed that there was no marked malignant progression observed after treatment with a CCR2 antagonist in their postsurgical recurrence animal models and that animals receiving CCR2 inhibition had a much longer survival rate, suggesting that CCL2 or CCR2 may be a potential targeting strategy in cancer treatment. However, its safety and efficacy need more clinical trial support.

The present study has certain limitations. Firstly, we only analyzed Snail, E-cadherin and vimentin expression. The absence of analysis of Slug as well as other epithelial and mesenchymal markers (N-cadherin and ZO-1), in addition to the absence of confocal analysis of E-cadherin and/or vimentin staining in the cells is one of the limitations of the present study. Secondly, despite that we detected Gli-1 expression via western blot analysis and immunofluorescence staining, the absence of Gli-1 reporter luciferase assays in response to CCL2 performed in order to demonstrate that there is an activation of the $\mathrm{Hh}$ pathway is another limitation of the present study.

In conclusion, we found that the CCL2/CCR2 axis induced $\mathrm{HCC}$ invasion and EMT in vitro through the activation of the Hh pathway. The CCL2/CCR2 axis induced invasion and tumor cell EMT through the increase of SMO and Gli-1 expression. Our results revealed that the link between CCR2 and the Hh pathway plays an important role in HCC progression. Therefore, the CCL2/CCR2 axis may represent a promising therapeutic target to prevent HCC progression (Fig. 7).

\section{References}

1. Siegel RL, Miller KD and Jemal A: Cancer statistics, 2015. CA Cancer J Clin 65: 5-29, 2015.

2. Chen W, Zheng R, Baade PD, Zhang S, Zeng H, Bray F, Jemal A, Yu XQ and He J: Cancer statistics in China, 2015. CA Cancer J Clin 66: 115-132, 2016.

3. Xu Q, Wang Z, Chen X, Duan W, Lei J, Zong L, Li X, Sheng L, Ma J, Han L, et al: Stromal-derived factor-1 $\alpha /$ CXCL12-CXCR4 chemotactic pathway promotes perineural invasion in pancreatic cancer. Oncotarget 6: 4717-4732, 2015.

4. Wang Z, Xie H, Zhou L, Liu Z, Fu H, Zhu Y, Xu L and Xu J: CCL2/CCR2 axis is associated with postoperative survival and recurrence of patients with non-metastatic clear-cell renal cell carcinoma. Oncotarget 7: 51525-51534, 2016.

5. Yang Y, Zhai C, Chang Y, Zhou L, Shi T, Tan C, Xu L and $\mathrm{Xu} \mathrm{J}$ : High expression of chemokine CCL2 is associated with recurrence after surgery in clear-cell renal cell carcinoma. Urol Oncol 34: 238.e19-238.e26, 2016.

6. Zhang J, Patel L and Pienta KJ: CC chemokine ligand 2 (CCL2) promotes prostate cancer tumorigenesis and metastasis. Cytokine Growth Factor Rev 21: 41-48, 2010.

7. Lim SY, Yuzhalin AE, Gordon-Weeks AN and Muschel RJ: Targeting the CCL2-CCR2 signaling axis in cancer metastasis. Oncotarget 7: 28697-28710, 2016.

8. Rao Q, Chen Y, Yeh CR, Ding J, Li L, Chang C and Yeh S Recruited mast cells in the tumor microenvironment enhance bladder cancer metastasis via modulation of ER $\beta / C C L 2 / C C R 2$ EMT/MMP9 signals. Oncotarget 7: 7842-7855, 2016.

9. Li X, Yao W, Yuan Y, Chen P, Li B, Li J, Chu R, Song H, Xie D, Jiang $X$ and Wang H: Targeting of tumour-infiltrating macrophages via CCL2/CCR2 signalling as a therapeutic strategy against hepatocellular carcinoma. Gut 66: 157-167, 2017.

10. Shih YT, Wang MC, Zhou J, Peng HH, Lee DY and Chiu JJ: Endothelial progenitors promote hepatocarcinoma intrahepatic metastasis through monocyte chemotactic protein-1 induction of microRNA-21. Gut 64: 1132-1147, 2015.

11. Li X, Wang Z, Ma Q, Xu Q, Liu H, Duan W, Lei J, Ma J, Wang X, Lv S, et al: Sonic hedgehog paracrine signaling activates stromal cells to promote perineural invasion in pancreatic cancer. Clin Cancer Res 20: 4326-4338, 2014. 
12. Li X, Ma Q, Xu Q, Liu H, Lei J, Duan W, Bhat K, Wang F, Wu E and Wang Z: SDF-1/CXCR4 signaling induces pancreatic cancer cell invasion and epithelial-mesenchymal transition in vitro through non-canonical activation of Hedgehog pathway. Cancer Lett 322: 169-176, 2012

13. Diao Y, Azatyan A, Rahman MF, Zhao C, Zhu J, Dahlman-Wright $\mathrm{K}$ and Zaphiropoulos PG: Blockade of the Hedgehog pathway downregulates estrogen receptor alpha signaling in breast cancer cells. Oncotarget 7: 71580-71593, 2016.

14. Wang Y, Han C, Lu L, Magliato S and Wu T: Hedgehog signaling pathway regulates autophagy in human hepatocellular carcinoma cells. Hepatology 58: 995-1010, 2013.

15. Villavicencio EH, Walterhouse DO and Iannaccone PM: The sonic hedgehog-patched-gli pathway in human development and disease. Am J Hum Genet 67: 1047-1054, 2000.

16. Lei J, Ma J, Ma Q, Li X, Liu H, Xu Q, Duan W, Sun Q, Xu J, Wu Z and Wu E: Hedgehog signaling regulates hypoxia induced epithelial to mesenchymal transition and invasion in pancreatic cancer cells via a ligand-independent manner. Mol Cancer 12: 66,2013

17. Zheng X, Zeng W, Gai X, Xu Q, Li C, Liang Z, Tuo H and Liu Q Role of the Hedgehog pathway in hepatocellular carcinoma (Review). Oncol Rep 30: 2020-2026, 2013.

18. Li Q, Liu Z, Xu M, Xue Y, Yao B, Dou C, Jia Y, Wang Y, Tu K, Zheng X and Yao Y: PCAF inhibits hepatocellular carcinoma metastasis by inhibition of epithelial-mesenchymal transition by targeting Gli-1. Cancer Lett 375: 190-198, 2016.

19. Schmittgen TD and Livak KJ: Analyzing real-time PCR data by the comparative C(T) method. Nat Protoc 3: 1101-1108, 2008.

20. An J, Xue Y, Long M, Zhang G, Zhang J and Su H: Targeting CCR2 with its antagonist suppresses viability, motility and invasion by downregulating MMP-9 expression in non-small cel lung cancer cells. Oncotarget 8: 39230-39240, 2017.

21. Thiery JP, Acloque H, Huang RY and Nieto MA: Epithelial-mesenchymal transitions in development and disease. Cell 139: 871-890, 2009.

22. Che L, Yuan YH, Jia J and Ren J: Activation of sonic hedgehog signaling pathway is an independent potential prognosis predictor in human hepatocellular carcinoma patients. Chin J Cancer Res 24: 323-331, 2012.

23. Kasper M, Regl G, Frischauf AM and Aberger F: GLI transcription factors: Mediators of oncogenic Hedgehog signalling. Eur J Cancer 42: 437-445, 2006.

24. Li Y, Yang W, Yang Q and Zhou S: Nuclear localization of GLI1 and elevated expression of FOXC2 in breast cancer is associated with the basal-like phenotype. Histol Histopathol 27 475-484, 2012

25. Chen JK, Taipale J, Cooper MK and Beachy PA: Inhibition of Hedgehog signaling by direct binding of cyclopamine to Smoothened. Genes Dev 16: 2743-2748, 2002.

26. Sahin H, Trautwein C and Wasmuth HE: Functional role of chemokines in liver disease models. Nat Rev Gastroenterol Hepatol 7: 682-690, 2010.

27. Mandrekar P, Ambade A, Lim A, Szabo G and Catalano D: An essential role for monocyte chemoattractant protein-1 in alcoholic liver injury: Regulation of proinflammatory cytokines and hepatic steatosis in mice. Hepatology 54: 2185-2197, 2011.

28. Baeck C, Wehr A, Karlmark KR, Heymann F, Vucur M, Gassler N, Huss S, Klussmann S, Eulberg D, Luedde T, et al: Pharmacological inhibition of the chemokine CCL2 (MCP-1) diminishes liver macrophage infiltration and steatohepatitis in chronic hepatic injury. Gut 61: 416-426, 2012.
29. Qian BZ, Li J, Zhang H, Kitamura T, Zhang J, Campion LR, Kaiser EA, Snyder LA and Pollard JW: CCL2 recruits inflammatory monocytes to facilitate breast-tumour metastasis. Nature 475: 222-225, 2011.

30. Huber MA, Kraut N and Beug H: Molecular requirements for epithelial-mesenchymal transition during tumor progression. Curr Opin Cell Biol 17: 548-558, 2005.

31. Wan Z, Pan H, Liu S, Zhu J, Qi W, Fu K, Zhao T and Liang J: Downregulation of SNAIL sensitizes hepatocellular carcinoma cells to TRAIL-induced apoptosis by regulating the NF- $\mathrm{BB}$ pathway. Oncol Rep 33: 1560-1566, 2015.

32. Zhang M, Dong X, Zhang D, Chen X and Zhu X: High expression of Snail and NF- $\kappa$ B predicts poor survival in Chinese hepatocellular carcinoma patients. Oncotarget 8: 4543-4548, 2017.

33. Lee CC, Ho HC, Su YC, Lee MS, Hung SK and Lin $\mathrm{CH}$ : MCP1-induced epithelial-mesenchymal transition in head and neck cancer by AKT activation. Anticancer Res 35: 3299-3306, 2015

34. Yang Z, Koehler AN and Wang L: A novel small molecule activator of nuclear receptor SHP inhibits HCC cell migration via suppressing Ccl2. Mol Cancer Ther 15: 2294-2301, 2016.

35. Wang J, Peng Y, Liu Y, Yang J, Huang M and Tan W: AT-101 inhibits hedgehog pathway activity and cancer growth. Cancer Chemother Pharmacol 76: 461-469, 2015

36. Mimeault M, Rachagani S, Muniyan S, Seshacharyulu P, Johansson SL, Datta K, Lin MF and Batra SK: Inhibition of hedgehog signaling improves the anti-carcinogenic effects of docetaxel in prostate cancer. Oncotarget 6: 3887-3903, 2015.

37. Zuo M, Rashid A, Churi C, Vauthey JN, Chang P, Li Y, Hung MC, Li D and Javle M: Novel therapeutic strategy targeting the Hedgehog signalling and mTOR pathways in biliary tract cancer. Br J Cancer 112: 1042-1051, 2015.

38. Huang S, He J, Zhang X, Bian Y, Yang L, Xie G, Zhang K, Tang W, Stelter AA, Wang Q, et al: Activation of the hedgehog pathway in human hepatocellular carcinomas. Carcinogenesis 27: 1334-1340, 2006

39. Chuang PT and McMahon AP: Vertebrate Hedgehog signalling modulated by induction of a Hedgehog-binding protein. Nature 397: 617-621, 1999.

40. Cai H, Li H, Li J, Li X, Li Y, Shi Y and Wang D: Sonic hedgehog signaling pathway mediates development of hepatocellular carcinoma. Tumour Biol 37: 16199-16205, 2016.

41. Huang XH, Chen JS, Wang Q, Chen XL, Wen L, Chen LZ, Bi J, Zhang LJ, Su Q and Zeng WT: miR-338-3p suppresses invasion of liver cancer cell by targeting smoothened. J Pathol 225: 463-472, 2011.

42. Pienta KJ, Machiels JP, Schrijvers D, Alekseev B, Shkolnik M, Crabb SJ, Li S, Seetharam S, Puchalski TA, Takimoto C, et al: Phase 2 study of carlumab (CNTO 888), a human monoclonal antibody against CC-chemokine ligand 2 (CCL2), in metastatic castration-resistant prostate cancer. Invest New Drugs 31: 760-768, 2013.

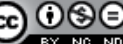

This work is licensed under a Creative Commons Attribution-NonCommercial-NoDerivatives 4.0 International (CC BY-NC-ND 4.0) License. 